\title{
Gas Chromatographic determination of parabens after derivatization and dispersive microextraction
}

\author{
V.M. Levchyk, M.F. Zui \\ Taras Shevchenko National University of Kyiv, 64/13, Volodymyrska Street, City of Kyiv, \\ Ukraine, 01601 \\ V_levchuk@univ.kiev.ua
}

\begin{abstract}
In this study a dispersive liquid-liquid microextraction method combines with in situ derivatization. The proposed method was used for the extraction and preconcentration of some preservatives including methylparaben, ethylparaben, propylparaben and butylparaben from water matrices. The extracted compounds were determined by gas chromatography - flame ionization detector. Derivatization of parabens was carried out using propionic anhydride. The effects of the extraction solvent type, extraction and acylation time, derivative agent's volume, temperature, $\mathrm{pH}$ and ionic strength of solution on the extraction efficiency were investigated. $50 \mu \mathrm{L}$ of chloroform and $500 \mu \mathrm{L}$ of acetonitrile was applied as a mixture of suitable extraction and dispersive solvents. The mixture is centrifuged for $3 \mathrm{~min}$ at $4000 \mathrm{rpm}$. $20 \mu \mathrm{L}$ of propionic anhydride is used for the derivatization of parabens in the concentration range of $0.05-1 \mathrm{mg} \mathrm{L}^{-1}$ within $2-3$ minutes. The optimum $\mathrm{pH}$ range was $8,0-9,0$. The limits of detection (LOD) were $0,003 \mathrm{mg} / \mathrm{L}$ for methyl-, ethylparabens; 0,002 mg/L for propyl-, butylparabens, relative standard deviations (RSDs) were in the range of $2.0-10.0 \%(\mathrm{n}=3, \mathrm{P}=95 \%)$. The method was applied to the analysis of the four parabens in water. For the analysis of the spiked samples, the recovery above $100 \%$ was obtained.
\end{abstract}

Key words: paraben, dispersive microextraction, derivatization, propionic anhydride

\section{Introduction}

Parabens are the esters of p-hydroxybenzoic acid. Due to their antiseptic and fungicidal properties they are widely used as preservatives in cosmetic, pharmaceutical and food products $[1,2]$. The most common are methyl-, ethyland propylparabens, whose concentration in the various products does not exceed $0.4 \%$, if there is one paraben, or $0.8 \%$ if they contain two or more parabens.

Contradictory opinions on the toxicity and carcinogenicity of parabens [3] and the possibility of coming them into the environment and the human body creates the nessecity for 
regular monitoring of content of parabens in environmental objects and the development of new methods of their identification, including extraction and preconcentration.

The traditional method of determining the parabens in pharmaceutical products and cosmetic products is high performance liquid chromatography (HPLC) with UV detector having a sensitivity of $5-10 \mathrm{mg} / \mathrm{L}$ [4], which allows to determine the content of these components in pharmaceutical and cosmetic products. However, these compounds may be presented in micro- and nanogram levels in biological and environmental samples. Modern method of sample preparation - microextraction is widely used for the separation and preconcentration of such quantities of parabens. According to the literature, methods for preconcentration of parabens, which are based on the singl-drop liquid microextraction combines with silylation [5], the dispersion liquid-liquid microextraction (DLLME) without [6] or with derivatization by acetic anhydride [7], followed by chromatographic determination are known. Despite the advantages of the developed techniques: simplicity, rapidity, the use of small amounts of toxic organic solvents, a wide range of detectable concentrations the disadvantage is the low stability of the system when using singdrop microextraction, low limit of detection for methylparaben when using dispersive microextraction. DLLME of acetyl derivatives is no effective for analysis solutions with a high different products, biological samples, content of inorganic salts (ionic strength of the solution $\geq 0,5$ ) [7], although such samples are widely used in pharmaceutical, biological and environmental samples.

In this work, a method based on DLLME combines with in situ derivatization and gas chromatographic detection equipped with a flame ionization detector (GC-FID) was developed for determination of parabens in water samples. Parabens are derivatizated by propionic anhydride. The effects of the extraction solvent type, extraction and acylation time, derivative agent's volume, temperature, $\mathrm{pH}$ and the ionic strength of the solution on the extraction efficiency were investigated. The analytical performance and possible application of the method in real sample analysis was investigated.

\section{Experimental part}

\section{Reagents}

Methyl-4-hydroxybenzoate (methylparaben) (99\%), ethyl-4-hydroxybenzoate (ethylparaben) (99\%), propyl-4-hydroxybenzoate (propylparaben) $(99 \%)$, butyl-4hydroxybenzoate (butylparaben) (99\%), chloroform $\quad\left(\mathrm{CHCl}_{3}\right) \quad(\geq 99 \%), \quad$ carbon tetrachloride $\quad\left(\mathrm{CCl}_{4}\right) \quad(\geq 99.5 \%), \quad 1,2-$ dichloromethane $\quad\left(\mathrm{CH}_{2} \mathrm{Cl}_{2}\right) \quad(\geq 99 \%)$ were purchased from Sigma-Aldrich (St. Louis, MO,USA), and $\mathrm{NaCl}$ (analytical grade), 
acetone, methanol, acetonitrile were purchased from "Reachim" (Ukraine).

Propionic anhydride ( $\geq 99 \%$, di-Potassium carbonate $\left(\mathrm{K}_{2} \mathrm{CO}_{3}\right)(\geq 99 \%)$ were purchased from Sigma-Aldrich (St.Louis, MO, USA).

A standard stock solution containing $1 \mathrm{mg} \mathrm{mL}^{-1}$ of methylparaben, ethylparaben, propylparaben and butylparaben was prepared on acetone. The stock solutions were refrigerated at $+4{ }^{\circ} \mathrm{C}$. Working standard solutions were prepared daily by diluting the stock standard solution with distilled water to required concentrations.

\section{Sample preparation}

The lake water was taken before the analysis. The lake water was taken in Kyiv, filtered through cellulose membrane filter with a pore size of $0.45 \mu \mathrm{m}$ and stored in a refrigerator at $4^{\circ} \mathrm{C}$.

\section{DLLME procedure}

Eight millilitres of aqueous solution of methylparaben, ethylparaben, propylparaben and butylparaben containing $0.02 \mathrm{~g} \mathrm{~mL}^{-1}$ of $\mathrm{K}_{2} \mathrm{CO}_{3}$ and $10 \mu \mathrm{L}$ of propionic anhydride was placed in a $12 \mathrm{~mL}$ centrifuge tube with a conic bottom; $0.57 \mathrm{~mL}$ of the solution containing 0.50 $\mathrm{mL}$ of acetonitrile (as disperser solvent), and 70 $\mu \mathrm{L}$ of chloroform (as extraction solvent) were rapidly injected to aqueous solution of parabens. The cloudy solution formed was centrifuged during $2 \mathrm{~min}$ at $5000 \mathrm{rpm}$. Chloroform phase with the analytes was sedimented on the bottom of the tube.

One milliliter of the extraction phase was taken using a $\quad 5 \quad \mu \mathrm{L}$ microsyringe (Agilent Technologies, USA) and injected into a gas chromatograph.

\section{GC analysis}

Gas chromatography was carried out in a Agilent Technologies 6890N (Agilent Technologies, USA) GC/FID coupled with an HP-5 fused silica capillary column $(30 \mathrm{~m} \times 0.32$ $\mathrm{mm}, 2.5 \mu \mathrm{m}$ film thickness) supplied by Agilent Technologies (Agilent Technologies, USA). The injector temperature was $270{ }^{\circ} \mathrm{C}$ and the detector temperature $300{ }^{\circ} \mathrm{C}$. The oven temperature was programmed, initially set at $130{ }^{\circ} \mathrm{C}$ for $2 \mathrm{~min}$, then gradually ramped to 230 ${ }^{\circ} \mathrm{C}\left(10^{\circ} \mathrm{C} \mathrm{min}^{-1}\right)$. The following gas flow rates were used: carrier (helium) 2, make-up gas (helium) 20, hydrogen 30 and air $300 \mathrm{~mL} \mathrm{~min}^{-1}$.

\section{Results and discussion}

\section{Derivatization Conditions}

Due to the low volatility and high polarity of target analytes for GC analysis, a derivatization step was needed to convert them into derivatives with better chromatographic properties. Also insample derivatization carried out before the extraction step can increase the extractability of the analytes. Among the different derivatization strategies (e.g., silylation, alkylation, acylation), in-situ acylation with anhydride of acetic or propionic acid is especially simple and fast. So, 
propionic anhydride was chosen in this work as a parabens derivatization agent. A chromatographic benefit of derivatization can be evidently seen in Fig. 1. Underivatized parabens produced asymmetric, broad peaks with significant tailing due to the interaction of hydroxyl groups with the chromatographic system. Derivatized paraben peaks are improved in the shapes, and were higher and narrower than underivatized paraben peaks.

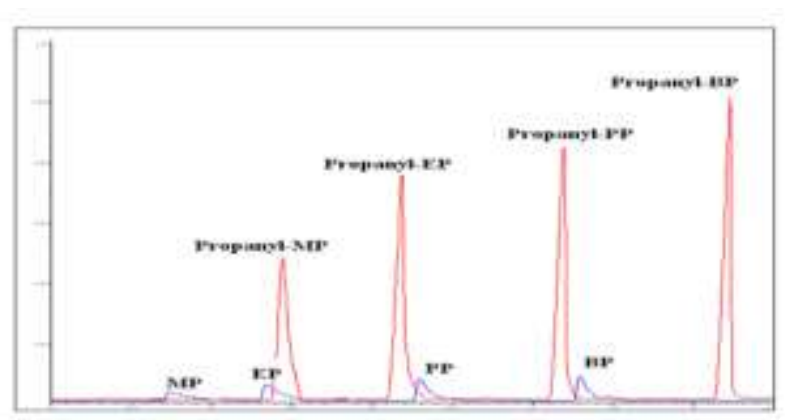

Fig. 1. Chromatogram peaks of parabens and propylparabens.

The effect of $\mathrm{pH}$ of the aqueous solution was studied in the range of 2 to 10. The data in Fig. 2 show that the formation of acyl derivatives of parabens is the most complete in the range of $\mathrm{pH} 8$ to 9. In the acidic, neutral and strongly alkaline environments chromatographic peak area of acylparabens decreases that can be caused by hydrolysis of the esters of phydroxybenzoic acid.

Extraction condition: sample volume, $8 \mathrm{~mL}$, concentration of parabens, $1.0 \mathrm{mg} / \mathrm{L}$, acetonitrile volume, $500 \mu$ l, chloroform volume, $70 \mu \mathrm{l}$, propionic anhydride volume, $20 \mu \mathrm{l}$

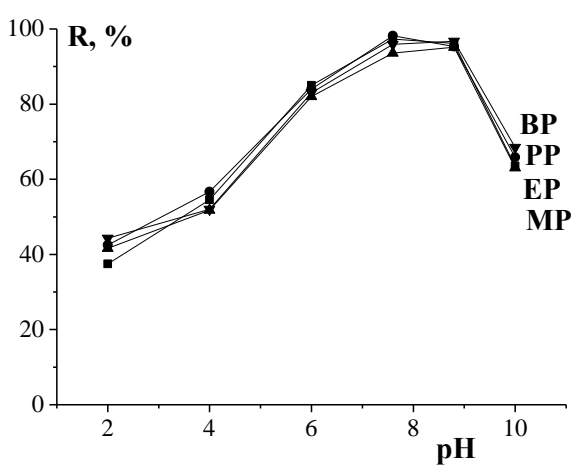

Fig. 2. Effect of $\mathrm{pH}$ on the derivatization efficiency of methyl (MP), ethyl (EP), propyl (PP), butylparabens (BP).

The quantity of propionic anhydride in situ derivatization step was optimized (Fig. 3). It was established that the full (100\%) acylation of parabens (no initial peaks of parabens in the chromatogram) was accomplished at molar ratio $30: 1$ of propionic anhydride to paraben.

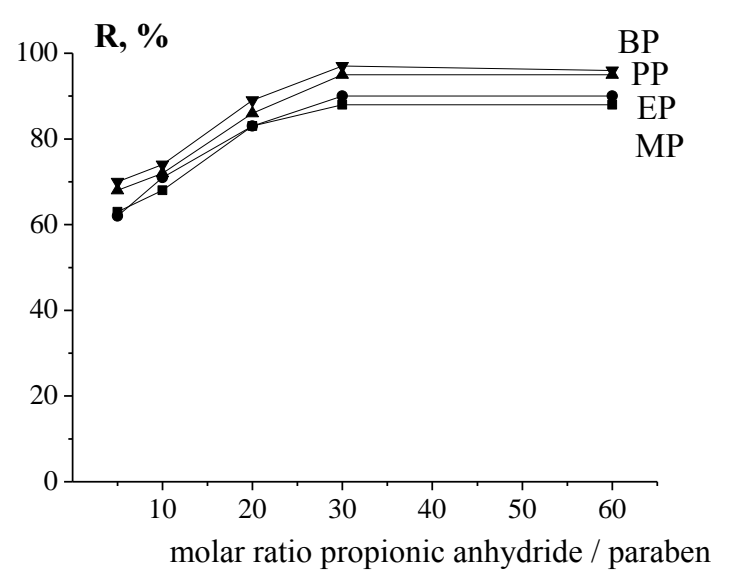

Fig. 3. Effect of molar ratio of propionic anhydride / paraben on the derivatization efficiency. 
Optimization of extraction time for DLLME with in situ derivatization method

One important parameter affecting DLLME with in situ derivatization was the extraction time. To optimize the extraction time, $1 \mathrm{mg} \mathrm{L}^{-1}$ standard solution of parabens was used. The extraction time profiles (equilibration curves) of parabens and the acyl parabens were determined by GC-FID. Parabens and the acyl derivatives of parabens reached equilibrium after $3 \mathrm{~min}$. Therefore, this time was used for the derivatization and preconcentration of parabens by DLLME in water samples.

\section{Study of the extraction solvent and disperser solvent}

The combination of the extraction solvent and the disperser solvent is a key issue in the DLLME process, and thus requires an exhaustive study prior to the final selection. On one hand, the extraction solvent should meet two basic properties, which are to extract the target analytes efficiently and to have low solubility in the aqueous solution. Moreover, organic phase should have dramatically higher density than water in order to remain in the bottom of the solution after centrifugation. On the other hand, the disperser solvent should be miscible in both aqueous sample and organic extraction solvent, and has also to form the cloudy solution. Also, after centrifugation, a phase separation has to be achieved. In this sense, dichloromethane (density $1.25 \mathrm{~g} \mathrm{~mL}^{-1}$, b.p. $40{ }^{\circ} \mathrm{C}$ ), chloroform (density $1.48 \mathrm{~g} \mathrm{~mL}^{-1}$, b.p. $62{ }^{\circ} \mathrm{C}$ ) and carbon tetrachloride (density $1.59 \mathrm{~g} \mathrm{~mL}^{-1}$, b.p. $\left.76.5{ }^{\circ} \mathrm{C}\right)$ were studied as extraction solvents, and acetone, acetonitrile and methanol were studied as disperser solvents. Therefore, all the possible combinations of extraction and disperser solvents were performed by injecting $1 \mathrm{~mL}$ of each disperser solvent containing $100 \mu \mathrm{L}$ of each extraction solvent to a $8 \mathrm{~mL}$ of an aqueous standard solution containing the four target analytes at $100 \mu \mathrm{g} \mathrm{L}^{-1}$. Using dichloromethane and carbon tetrachloride as extraction solvents, the extraction efficiency, $\mathrm{R}$ was lower than using chloroform. Results shown in Fig. 4 can be concluded that the best results were accomplished when acetonitrile was used as the disperser solvent and chloroform as the extraction solvent. Therefore, the mixture acetonitrile - chloroform was selected for further experiments.

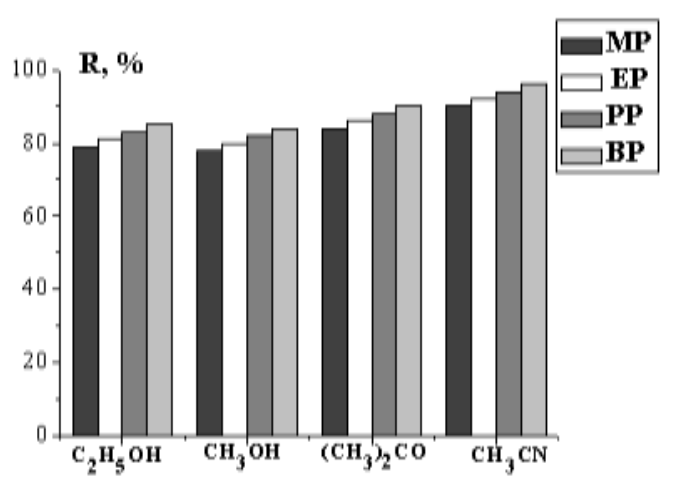

Fig. 4. Effect of disperser solvent on the extraction efficiency. Extraction conditions: sample volume, $8 \mathrm{~mL}$, concentration of parabens, $1.0 \mathrm{mg} / \mathrm{L}$; propionic anhydride 
volume, $20 \mu \mathrm{l}$; disperser volume, $500 \mu \mathrm{l}$, extraction solvent volume $70 \mu \mathrm{l}$.

\section{Effect of the extraction and disperser solvent} volume

The volume of chloroform was optimized by taking different volumes of chloroform ranges $40-100 \mu \mathrm{l}$ with acetonitrile $(500 \mu \mathrm{l})$ as disperser solvent and propionic anhydride as derivative reagent. The extraction efficiency, $\mathrm{R}$ of parabens was increased when there was used $70 \mu \mathrm{l}$ of chloroform (Fig. 5). Therefore, $70 \mu \mathrm{l}$ of chloroform was selected for further experiments.

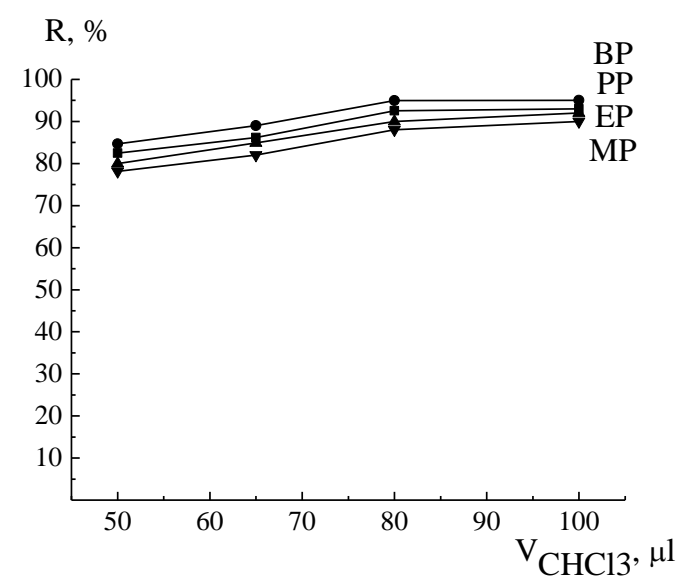

Fig. 5. Effect of extraction solvent volume on the extraction efficiency. Extraction conditions: sample volume, $8 \mathrm{~mL}$, concentration of parabens, $1.0 \mathrm{mg} / \mathrm{L}$; propionic anhydride volume, $20 \mu \mathrm{l}$; acetonitrile volume, $500 \mu \mathrm{l}$.

To investigate the effect of the disperser solvent volume, different acetonitrile volumes $(0.15-$ $0.9 \mathrm{~mL}$ ) and $70 \mu \mathrm{L}$ of extraction solvent were used. Due to increasing the acetonitrile volume, the extraction efficiency, $R$ initially enlarged (Fig. 6). At a low acetonitrile volume, the cloudy state was not stable, and probably this caused incomplete extraction. On the other hand, when the acetonitrile volume exceeded $0.5 \mathrm{~mL}$, the solubility of the parabens in water acetonitrile mixture increased and their concentration in sedimented phase decreased. According to the results, $0.4-0.7 \mathrm{~mL}$ of acetonitrile is optimal. So, a convenient volume of $0.5 \mathrm{~mL}$ acetonitrile and optimum chloroform volume $70 \mu \mathrm{L}$ were selected for the further work.

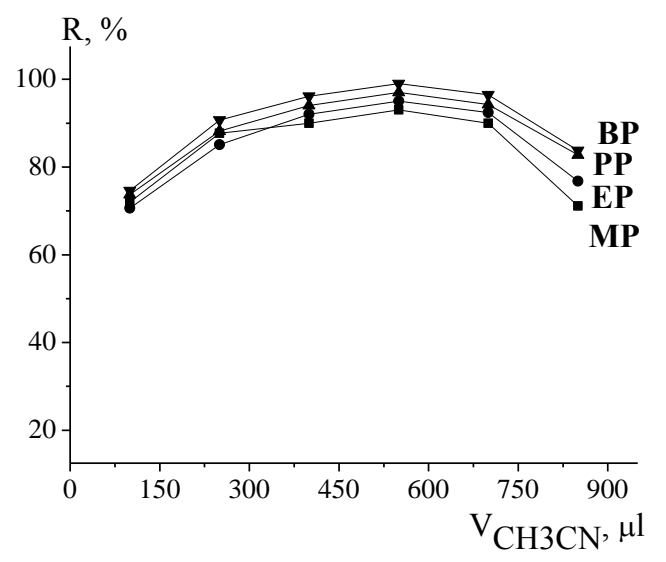

Fig. 6. Effect of disperser volume on the extraction efficiency. Extraction conditions: sample volume, $8 \mathrm{~mL}$, concentration of parabens, $1.0 \mathrm{mg} / \mathrm{L}$; propionic anhydride volume, $20 \mu \mathrm{l}$; chloroform volume, $70 \mu \mathrm{l}$.

\section{Effect of the ionic strength}

In general terms, the addition of salt improves the extraction as a result of the so-called saltingout effect, because the presence of salt reduces the solubility of the organic compounds in water 
and forces them to pass to the extraction phase. Thus, in order to study this effect, $\mathrm{NaCl}$ was added to the donor aqueous phase at different concentrations up to $15 \%(\mathrm{~m} / \mathrm{v})$. Results revealed that, salt addition had effect on the extraction efficiency of DLLME (Fig. 7). Therefore, $3 \%$ salt was added for further experiments.

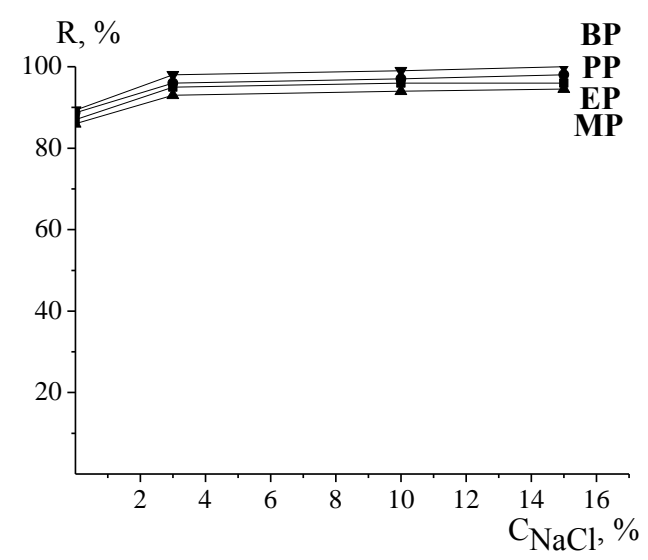

Fig. 7. Effect of $\mathrm{NaCl}$ concentration on the extraction efficiency. Extraction condition: sample volume, $8 \mathrm{~mL}$, concentration of parabens, $1.0 \mathrm{mg} / \mathrm{L}$, propionic anhydride volume, $20 \mu \mathrm{l}$; acetonitrile volume, $500 \mu \mathrm{l}$., chloroform volume, $70 \mu 1$.

\section{Determination of parabens in lake water samples}

In this study, lake (Kyiv, Ukraine) water sample was analyzed for parabens using the present method. The results showed that the lake all water sample analyzed was free of parabens.

To assess matrix effect, $8 \mathrm{~mL}$ of lake water were spiked with $50-200 \mu \mathrm{g} \mathrm{L}^{-1}$ of parabens. Concentrations of parabens were calculated using calibration curves obtained in distilled water and were controlled by method of standard additions. Relative recoveries were determined as the ratio of the concentrations found in real and distilled water samples spiked at the same analyte concentrations. The data of the analysis demonstrate little matrix effect on DLLME with the recoveries 96.7 - 99.5, \%.

Table 1. Recoveries of spiked parabens in model solution and lake water $(\mathrm{n}=3, \mathrm{P}=95 \%)$

\begin{tabular}{|l|l|l|l|l|l|}
\hline $\begin{array}{l}\text { Ana- } \\
\text { lyte }\end{array}$ & $\begin{array}{l}\text { Spiked } \\
\text { Level, } \\
\text { mg/L }\end{array}$ & \multicolumn{2}{|l|}{$\begin{array}{l}\text { Model solution } \\
\text { Calibration curve } \\
\text { method }\end{array}$} & $\begin{array}{l}\text { Lake water } \\
\text { Calibration curve } \\
\text { method }\end{array}$ \\
\cline { 3 - 6 } & $\begin{array}{l}\text { Recovery, } \\
\%\end{array}$ & $\begin{array}{l}\text { RSD, } \\
\%\end{array}$ & $\begin{array}{l}\text { Recovery, } \\
\%\end{array}$ & $\begin{array}{l}\text { RSD, } \\
\%\end{array}$ \\
\hline MP & 0.05 & 98,8 & 6.3 & 96.7 & 4.4 \\
& 0.20 & 99.1 & 3.1 & 98.2 & 3.8 \\
\hline EP & 0.05 & 96.2 & 5.3 & 98.2 & 5.4 \\
& 0.20 & 98.3 & 4.5 & 99.5 & 3.8 \\
\hline PP & 0.05 & 98.3 & 4.8 & 97.9 & 6.7 \\
& 0.20 & 98.9 & 3.0 & 99.1 & 3.7 \\
\hline BP & 0.05 & 97.6 & 8.0 & 98.0 & 6.7 \\
& 0.20 & 98.8 & 6.0 & 100.0 & 5.6 \\
\hline
\end{tabular}

The quantity parameters of the suggested method such as limit of detection, enrichment factor, and repeatability were calculated under the optimized extraction conditions. The results are presented in Table 2. These data show that repeatability of the method is satisfactory.

Table 2. Enrichment factors, repeatabilities and detection limits of proposed method of preconcentration and GC determination of parabens

\begin{tabular}{|c|c|c|c|}
\hline Analyte & $\begin{array}{c}\text { Enrichment } \\
\text { factor }\end{array}$ & $\mathrm{RSD}, \%$ & $\begin{array}{c}\mathrm{LOD}, \\
\mathrm{mg} / \mathrm{L}\end{array}$ \\
\hline MP & 280 & 4.1 & 0,003 \\
\hline
\end{tabular}


FRENCH-UKRAINIAN JOURNAL OF CHEMISTRY (2015, VOLUME 03, ISSUE 02)

\begin{tabular}{|l|l|l|l|}
\hline EP & 290 & 3.1 & 0,002 \\
\hline PP & 300 & 2.5 & 0,002 \\
\hline BP & 320 & 2.7 & 0,002 \\
\hline
\end{tabular}

\section{Conclusion}

The method of dispersive liquid-liquid microextraction of derivatized parabens from water samples followed with gas chromatographic determination has been developed. For the derivatization of parabens, acylation with propionic anhydride has been applied. The proposed method provides high enrichment factors, it is compatible with GC, it is precise, reproducible and linear over a broad concentration range, and is environmentel friendly. Detection limits were three to nine times smaller than those obtained for underivatized parabens using the same GC equipment. Detection limits can be additionally reduced using mass spectrometric detection instead of flame ionization detection.

\section{References}

1. J.A.Ocaña-González, M.Villar-Navarro, M.Ramos-Payán et al. Analyt. Chim. Acta. 2015, 858, 1-15. doi: $\underline{10.1016 / j . a c a .2014 .07 .002 .}$.

2. I.Baranowska, I.Wojciechowska. Pol. J. Environ. Study. 2013, 22 (6), 1609 - 1625.

3. Scientific Committee on consumer safety (SCCS). Opinion on parabens. COLIPA $\mathrm{n}^{\mathrm{o}} \mathrm{P} 82$. SCCS/1514/13. 2013, 3 May, 1 - 50. doi: $10.2772 / 66369$.

4. Ascentis express. 2012. Sigma-Aldrich. LCGC North America. Solutions for separation 